\title{
Composite Fermion Description of the Excitations of the Paired Pfaffian Fractional Quantum Hall State
}

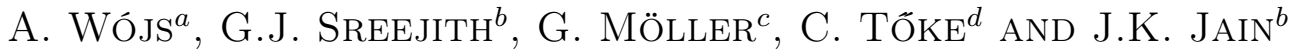 \\ ${ }^{a}$ Institute of Physics, Wrocław University of Technology, Wybrzeże Wyspiańskiego 27, 50-370 Wrocław, Poland \\ ${ }^{b}$ Department of Physics, 104 Davey Lab, Pennsylvania State University, University Park, PA 16802, USA \\ ${ }^{c}$ TCM Group, Cavendish Laboratory, J.J. Thomson Av., Cambridge CB3 0HE, United Kingdom \\ ${ }^{d}$ Institute of Physics, University of Pécs, 7624 Pécs, Hungary
}

\begin{abstract}
We review the recently developed bipartite composite fermion model for the excitations of the so-called Pfaffian state, a promising model for the fractional quantum Hall effect at the Landau level filling factor $\nu=5 / 2$. In particular, we demonstrate a necessary ingredient of the non-Abelian braid statistics, namely the degeneracy of spatially indistinguishable configurations of widely separated quasiparticles.
\end{abstract}

PACS: 73.43.-f, 05.30.Pr, 71.10.Pm

\section{Introduction}

The fractional quantum Hall effect [1] is a macroscopic manifestation of the formation of a new phase of matter in quasi-two-dimensional systems of interacting electrons exposed to a high perpendicular magnetic field $B[2]$. The phenomenon consists of the simultaneous quantization of the perpendicular (Hall) resistivity $\rho_{x y}$ and the vanishing of the longitudinal resistivity $\rho_{x x}$. It occurs when electrons fill a particular fraction $\nu_{n}=1 / 3,2 / 5$, etc., of a massively degenerate Landau level (labeled by $n=0,1, \ldots)$, which is effectively decoupled from all other levels because of the large cyclotron energy separation $h \omega_{\mathrm{c}} \propto B$, exceeding the characteristic interaction (Coulomb) energy $e^{2} / \lambda \propto B^{1 / 2}$, defined in terms of magnetic length scale $\lambda=(\hbar c / e B)^{1 / 2}$.

The family of incompressible electron liquids occurring in the lowest Landau level $(n=0)$ is well understood in terms of certain novel particles called "composite fermions" (CFs) [3], which are intuitively viewed as bound states of electric charge and magnetic flux specifically, as bound states of electrons and an even number $2 p$ of magnetic flux quanta $\phi_{0}=h c / e$. They move in an appropriately reduced effective magnetic field $B^{*}=B-2 p \phi_{0} \varsigma$, where $\varsigma$ is the electron concentration (simply connected to the filling factor via $\nu B=\phi_{0} \varsigma$ or $\nu=2 \pi \lambda^{2} \varsigma$ ), and interact through greatly reduced residual forces. The effective field $B^{*}$ can be converted to an effective filling factor of the composite fermions, $\nu^{*}=\left(\nu^{-1}-2 p\right)^{-1}$. More precisely, the composite fermions are defined as bound states of the electrons and pairs of vortices of the many-electron wave function, which (for $N$ electrons in the lowest Landau level of degeneracy $N_{\phi}=\Phi / \phi_{0}$, where $\Phi=B A$ is the total mag- netic flux through a sample of area $A$, in the symmetric gauge) has the form of an antisymmetric polynomial in the complex electronic coordinates $z=x+\mathrm{i} y$, of a fixed degree $N_{\phi}$, multiplied by a symmetric exponential tail. The emergence of weakly interacting composite fermions in a system of strongly interacting quasi-two-dimensional electrons in the Hilbert space severely restricted (as a result of the single-particle Landau quantization) to an isolated Landau level is thus equivalent to specifying a particular form of electronic correlation, given by a simple Jastrow prefactor of power $2 p$ in the many-electron wave function.

The composite fermion theory gives an elegant and intuitive understanding of the otherwise unexpected incompressibility observed experimentally at a (universal) series of filling factors $\nu=q /(2 p q+1)$, in terms of the non-interacting composite fermions at integral fillings $\nu^{*}=q$ of the Landau levels, and thus forming a unique ground state separated from the continuum of excitations by an effective composite fermion cyclotron gap $h \omega_{\mathrm{c}}^{*}$. The two kinds of quasiparticles - positive quasiholes (QHs) and negative quasielectrons (QEs), formed in an underlying incompressible electron liquid as a result of insertion or removal of a magnetic flux quantum - correspond to the vacancies in the otherwise full $n^{*}=q$ composite fermion level or to the few composite fermions in the otherwise empty level $n^{*}=q+1$. Furthermore, the collective neutral modes correspond to excitonic states of $\mathrm{QE}+\mathrm{QH}$ pairs.

The accuracy of the composite fermion predictions is illustrated in Figs. 1 and 2, showing several examples of energy spectra, calculated on a sphere [4] for fairly large finite systems representing a selection of different filling factors $\nu$. In this geometry, electrons are confined 
to the surface of a unit sphere, the Coulomb interaction potential is taken proportional to the inverse chord distance, and the radial magnetic field is produced by a Dirac monopole of the quantized strength $\Phi=2 Q h \mathrm{c} / \mathrm{e}$, yielding the magnetic length $\lambda=Q^{-1 / 2}$ (relative to the unit radius) and the Landau level degeneracy of $2 Q+1$ ( $n$-th Landau level appearing in the form of an angular momentum shell with $\ell=Q+n)$. The many-electron spectra are then obtained in the form of dependence of (Coulomb) energy $E$ as a function of total orbital angular momentum $L$ (the conserved orbital quantum number on a sphere).
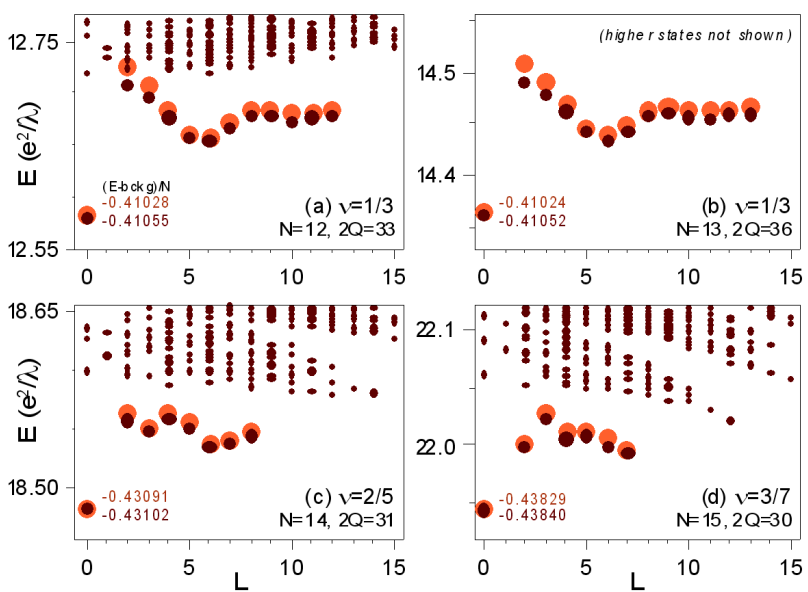

Fig. 1. Demonstration of the accuracy of the composite fermion prediction of the Coulomb energy spectra in the partially filled lowest Landau level, calculated in the spherical geometry for fairly large numbers of electrons $N$ and the magnetic monopole strengths $2 Q$ corresponding to the indicated filling factors $\nu=1 / 3$ (here, two different sizes; only the lowest states shown at each angular momentum $L$ for $N=13$ ), $2 / 5$, and $3 / 7$, corresponding to (respectively) $q=1,2$, and 3 Landau levels filled by the composite fermions each carrying $2 p=2$ magnetic flux quanta. The darker dots are the Coulomb eigenenergies (obtained from exact numerical diagonalization of the $N$-body Coulomb Hamiltonians) and the brighter ones are the Coulomb energies of the corresponding composite fermion wave functions. The states shown with larger dots are the nondegenerate $(L=0)$ ground states and the "magneto-roton" collective modes (with $L>0$ representing quantized wave vectors). The pairs of numbers indicated for each ground state are the (exact Coulomb and composite fermion trial) correlation energies per particle, obtained from $E / N$ by subtraction of the electrostatic contribution from the charge compensating background, $(\mathrm{Ne})^{2} / 2$.

The composite fermion theory also predicts the exact form of the many-electron wave functions (appropriate polynomials) describing both the ground states and their excitations. The composite fermion wave functions are generally constructed as

$$
\mathcal{P}_{\mathrm{LLL}} \mathcal{D}^{2 p} \Psi_{q} \equiv \mathcal{P}_{\mathrm{LLL}} \prod\left(z_{i}-z_{j}\right)^{2 p} \Psi_{q}\left(z_{1}, z_{2}, \ldots\right),
$$

where $\Psi_{q}$ is a Slater determinant many-fermion wave

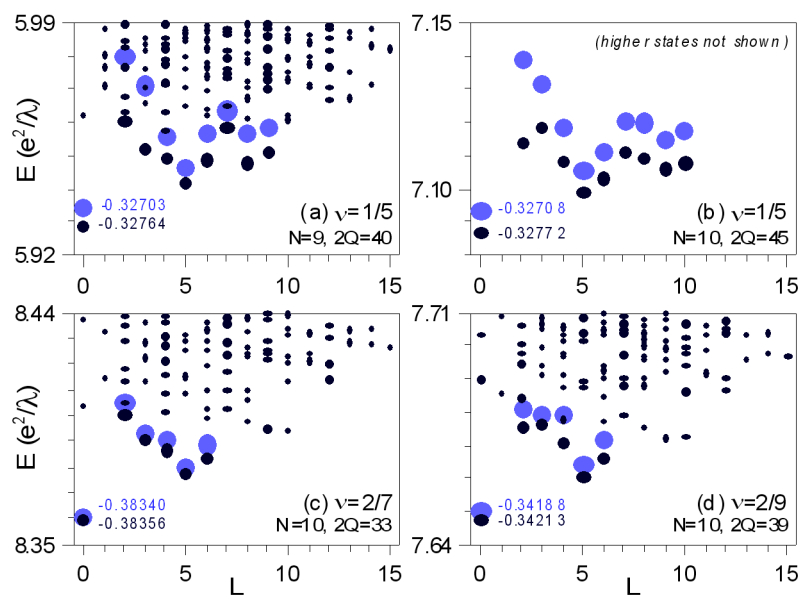

Fig. 2. The same as Fig. 1, but for the composite fermions carrying $2 p=4$ magnetic flux quanta, corresponding to the filling factors $\nu=1 / 5,2 / 7$, and $2 / 9$, or (respectively) to $q=1,-2$, and 2 composite fermion Landau levels (negative $q$ means that the effective magnetic flux $2 Q^{*}$ is oriented opposite to the original flux $2 Q$ ).

function describing $q$ filled Landau levels, possibly with a number of additional vacancies in the level $q$ and/or particles in the level $q+1, \mathcal{D}^{2 p}$ is the Jastrow correlation factor, and $\mathcal{P}_{\text {LLL }}$ projects the wave function into the lowest Landau level. The exponential tail $\exp \left(-\sum\left(\left|z_{i}\right| / 2 \lambda\right)^{2}\right)$ is identical for each polynomial, so it is omitted here for simplicity. Such wave functions were used in Figs. 1 and 2 for comparison with the exact eigenstates of the Coulomb interaction.

The accumulated wealth of successful tests (numerical and experimental) have established composite fermions as a secure framework for our understanding of fractional quantum Hall effect in the lowest Landau level.

\section{Topological degeneracy, non-Abelian statistics}

The quasiparticles of incompressible electron liquids in the lowest Landau level, which in the composite fermion theory are represented by the vacancies or particles in the almost full or almost empty composite fermion levels, have fractional charge excess or deficiency associated with them $( \pm e / q)$ and are believed to obey Abelian braid statistics. More exotic many-electron wave functions, describing ground states with quasiparticle excitations obeying non-Abelian braid statistics, have been proposed within the conformal field theories corresponding to the sets of fields with multi-valued fusion rules. The simplest one is called the $Z_{2}$ or the Ising theory, which contains one boson "vacuum" field $\mathbf{1}$, one fermion "electron" field $\psi$, and an additional half-fermion "vortex" field $\sigma$, with the following set of fusion rules [5]:

$$
\psi \times \psi=\mathbf{1}, \sigma \times \psi=\sigma, \sigma \times \sigma=1+\psi,
$$

which say that a pair of fermions is equivalent to a bo- 
son, and a vortex with a fermion together behave as a vortex, but two vortices may fuse either to a boson or a fermion, giving rise to a multiply degenerate space for states with specified positions of vortices. This topological degeneracy first occurs for as many as four vortices, which together can fuse to a boson in two distinct ways - either fusing in pairs to two fermions or to two bosons:

$$
\mathbf{1}=(\sigma \times \sigma) \times(\sigma \times \sigma)=\mathbf{1} \times \mathbf{1}+\psi \times \psi .
$$

Such topological degeneracy, allowing for multiple quantum states representing an identical spatial configuration of four (or more) vortices, opens a possibility for non-Abelian braid statistics. Indeed, the braids in such a multi-dimensional subspace of identical configurations require multi-dimensional (matrix) representation, which is inherently non-commutative.

The physical realization of the non-Abelian anyon braid statistics is an exciting prospect on its own conceptual grounds. However, it has also received attention because of a proposal [6] to employ the topologically degenerate states and their braiding in the encoding and manipulation of quantum information which, due to the inherent protection of such qubits from any local disturbances (such as phonons or nuclear spins), would have immunity from decoherence.

\section{Pfaffian many-electron wave function}

The simplest many-electron wave function emerging from the Ising conformal field theory is the so-called Pfaffian state [7] describing a particular form of electron correlation in a half-filled Landau level $(\nu=1 / 2)$ :

$$
\Psi_{\mathrm{Pf}}=\mathcal{D}^{2} \operatorname{Pf}\left(\frac{1}{z_{i}-z_{j}}\right) \equiv \prod\left(z_{i}-z_{j}\right)^{2} \operatorname{Pf}\left(\frac{1}{z_{i}-z_{j}}\right),
$$

where $\operatorname{Pf}\left(a_{i j}\right)$ denotes the Pfaffian of a skew-symmetric, even-dimensional matrix $a$. Writing it explicitly up to the normalization constant,

$$
\operatorname{Pf}\left(a_{i j}\right) \equiv \mathcal{A}\left(a_{12} a_{34} a_{56} \ldots\right),
$$

where the operator $\mathcal{A}$ denotes antisymmetrization over all indices. It is clear that $\Psi_{\mathrm{Pf}}$ describes a paired state of composite fermions (as ensured by the Pfaffian and Jastrow factors, respectively).

The above Pfaffian wave function represents a unique zero-energy ground state of a particular three-body model Hamiltonian $\mathcal{H}_{\mathrm{Pf}}$ which in real space take the form of the three-body contact repulsion and inside a Landau level corresponds to a simple triplet pseudopotential with the sole non-vanishing (positive) coefficient at the minimum allowed three-body relative angular momentum, $m=3$. (This is reminiscent of the Laughlin $\nu=1 / 3$ state - a unique zero-energy ground state of the pair contact repulsion.)

Quasiholes are created in the Pfaffian ground state in pairs for each additional flux quantum, and thus each of them carries a fractional charge $e / 4$. They offer a particular example of non-Abelian anyons. To be precise, the space of configurations of $2 K$ localized and distant quasiholes is $2^{K-1}$-fold degenerate, as predicted from the Ising fusion rules (2).

These features are best illustrated in the exact energy spectra of the three-body repulsion $\mathcal{H}_{\mathrm{Pf}}$, as shown on two examples in Fig. 3. On a sphere, the Pfaffian ground state occurs at $2 Q=2 N-3$, as shown in frame (a) for $N=14$. Increasing flux by one, to $2 Q=2 N-2$, produces a pair of Pfaffian quasiholes which (not shown) results in a band of zero-energy states at $L=N / 2, N / 2-2, \ldots$, consistent with the addition rule for two equal angular momenta. Inserting two flux quanta to the ground state, to $2 Q=2 N-1(\mathrm{~b})$, produces a large number of zero-energy states corresponding to four quasiholes, whose counting is not consistent with Abelian statistics, instead showing an additional topological (exchange) degeneracy.
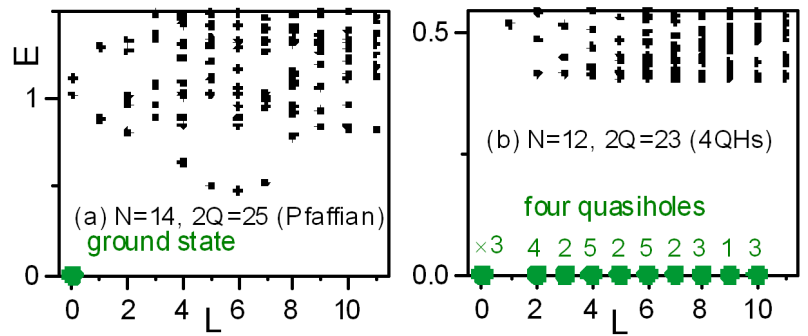

Fig. 3. The low-energy spectra of the model three-body "contact repulsion" Hamiltonian $\mathcal{H}_{\mathrm{Pf}}$ calculated by exact diagonalization on a sphere at two different combinations of the fermion number $N$ and magnetic flux $2 Q$, corresponding to (a) the Pfaffian $\nu=1 / 2$ ground state, and (b) two additional magnetic flux quanta inserted to such a ground state. The green labels in (b) give the degeneracy of zero-energy levels interpreted as four non-Abelian anyon quasiholes in the Pfaffian state of $N=12$ fermions, consistent with the prediction of the underlying Ising model.

The interpretation of the Pfaffian wave function (4) in terms of composite fermion pairing can be expressed rather intuitively in form of the following sequence of remarkable (though yet unproven) emergences:

(i) As a result of the Coulomb repulsion within a Landau level, each electron captures two vortices to become a composite fermion.

(ii) Because the Landau level is half-filled, the effective magnetic field $B^{*}$ vanishes, and the composite fermions form a two-dimensional, spin-polarized (due to the Zeeman effect governed by the original field $B$ ) Fermi sphere.

(iii) In the absence of a single-particle energy gap, the residual interaction among the composite fermions may (depending on sign) cause instability of the Fermi sphere through the Cooper pairing of the composite fermions.

(iv) The energy gap for breaking a pair in the resulting composite fermion superfluid causes incompressibility and fractional quantum Hall effect. 


\section{Pfaffian state in a half-filled Landau level}

The key question is whether the Pfaffian state (with its exotic excitations) provides a valid description of the Coulomb ground state. The fractional quantum Hall measurements at $\nu=1 / 2$ (i.e., at half-filling of the lowest Landau level) are consistent with a compressible phase, confirmed to be the Fermi sea of composite fermions. This suggests that the ground state is different from the Pfaffian, presumably because the residual composite fermion interaction invoked in the above point (iii) is insufficient to induce a pairing transition in the Fermi sea of composite fermions.

However, the analogous experiments at $\nu=5 / 2$ show clear signatures of quantized Hall effect [8]. This implies emergence of an incompressible ground state in the half-filled second $(n=1)$ Landau level - the counting $\nu=2+1 / 2$ reflecting the double spin degeneracy of the filled lowest $(n=0)$ level. The crucial question then becomes whether the $\nu=5 / 2$ quantum Hall state is equivalent to the Pfaffian state. Of course, the equivalence does not require that the actual Coulomb wave function be very close to the Pfaffian wave function (although that would definitely help). It would be sufficient to show adiabatic continuity between both ground states [9] and their excitations. This includes identity of such qualitative features as the spatial uniformity (non-degeneracy), full spin polarization of the ground state [10], an excitation gap, the particular electric charge $( \pm e / 4)$ and the braid properties (non-Abelian Ising anyons) of the quasiparticle excitations, or the number of collective modes. Further topologically protected features (preserved under continuous deformation) may include certain dynamical degeneracies, following from $p$-wave pairing or from fusion rules.

Let us note that the holonomic form of the Pfaffian wave function describes a many-electron state exclusively
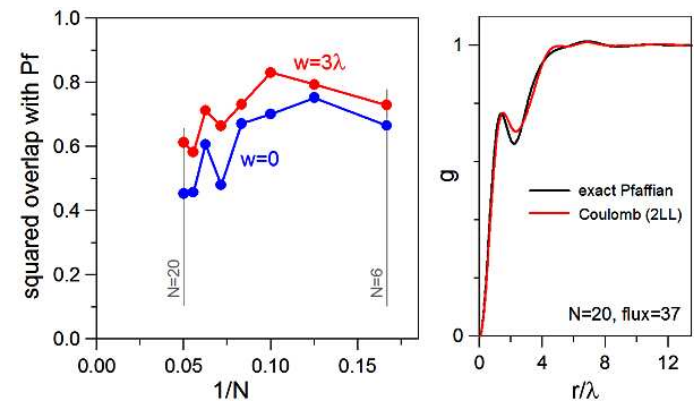

Fig. 4. Comparison of the Coulomb ground states in the half-filled second Landau level $(\nu=5 / 2)$ with the exact Pfaffian state, in the spherical geometry. Left: dependence of the squared overlaps on the electron number $N=6$ to 20 , for the layer widths $w=0$ and $3 \lambda$ (three magnetic lengths). Right: comparison of the pair-correlation functions $g(r)$ for $N=20$ and the flux $2 Q=2 N-3=37$. Evidently, positive identification of the $\nu=5 / 2$ state with the Pfaffian state based solely on such analysis is problematic. in the lowest Landau level. However, the problem of electrons in the second Landau level interacting with the Coulomb interaction can be mapped into that of electrons in the lowest Landau level with an effective interaction (using the concept of guiding-center positions), allowing an exact simulation of the $5 / 2$ problem in the lowest Landau level.

A comparison of the Pfaffian wave function with the actual $\nu=5 / 2$ state (the Coulomb ground state in the half-filled second Landau level) is illustrated in Fig. 4, showing the overlaps (as a function of electron number $N$ ) and comparing the pair-correlation functions (for a fairly large system, with $N=20$ ).

\section{Collective modes of the Pfaffian state}

An important aspect of the Pfaffian dynamics is that if composite fermions indeed form a $p$-wave paired state at $\nu=5 / 2$, then, in analogy to superconductivity, one can

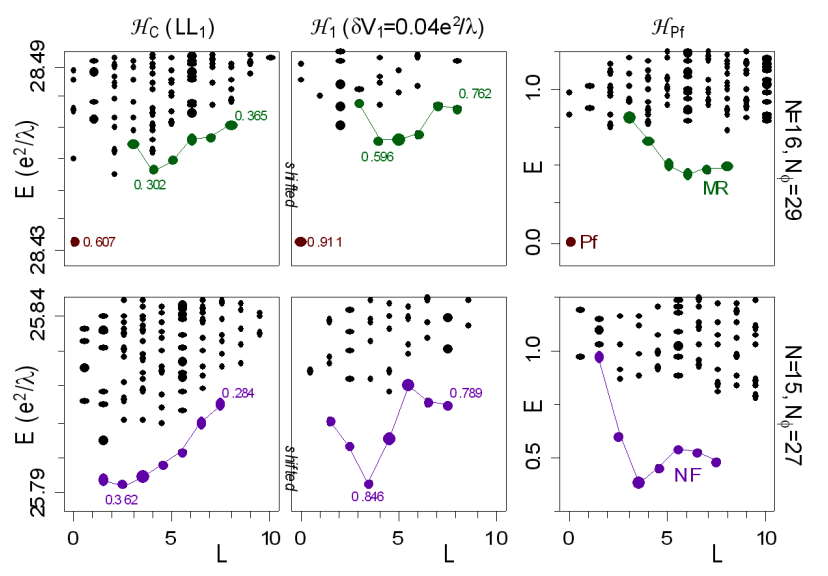

Fig. 5. The energy spectra of an even and odd number of fermions, $N=16$ and 15, on a sphere, at the Landau level degeneracy $N_{\phi}+1$ corresponding to the magnetic flux $2 Q \equiv N_{\phi}=2 N-3$, for three different Hamiltonians: $\mathcal{H}_{\mathrm{C}}$ is the Coulomb interaction in the second Landau level $(n=1), \mathcal{H}_{1}$ is the same Coulomb interaction but with an additional enhancement of the leading pair pseudopotential (at $m=1$ ) by the value $\delta V_{1}=0.04 e^{2} / \lambda$ which maximizes the overlap of the ground state with the Pfaffian state, and $\mathcal{H}_{\mathrm{Pf}}$ is the three-body contact repulsion for which the exact Pfaffian state is a unique zero-energy ground state. The low-lying states at the angular momenta $L$ predicted for the magneto-roton and unpaired-CF excitations are connected with lines, and their squared overlaps with the corresponding eigenstates of $\mathcal{H}_{\mathrm{Pf}}$ are indicated. The emergence of the pair of dispersions is especially clear for $\mathcal{H}_{\mathrm{Pf}}$ and $\mathcal{H}_{1}$. Graphs for $N=15$ were earlier presented in Ref. [11].

expect two kinds of collective modes: magneto-rotons (known from the fractional quantum Hall states in the lowest Landau level and representing excitons of composite fermions) and "unpaired composite fermions", also known as "neutral fermions". The latter are studied conveniently by considering systems with an odd number of 
electrons, which necessarily contain an unpaired fermion. The dispersions of both these modes has been studied recently by exact diagonalization [11, 12], in sufficiently large systems to identify a number of significant features. The examples of energy spectra for an even and odd electron number $N$, each one featuring one of the modes, are shown in Fig. 5.

When the low lying-states of the spectra obtained for different $N$ and interpreted as either the magneto-roton or the unpaired CF collective excitations are plotted together as a function of the wave vector $k$ (for the charge neutral modes moving along the great circles $k=L / R$ is trivially connected to the sphere radius $R \equiv 1$ and orbital angular momentum $L$ ), they produce well-defined curves representing a pair of continuous energy dispersions.

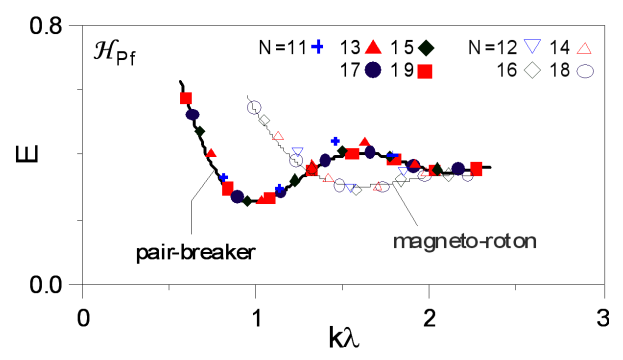

Fig. 6. Continuous dispersions of the magneto-roton and unpaired CF (or neutral fermion) collective excitations in the Pfaffian ground state, obtained by overlaying the discrete energy spectra of repulsive three-body Pfaffian Hamiltonian $\mathcal{H}_{\mathrm{Pf}}$ for different fermion numbers $N . k$ is the wave vector (proportional to angular momentum $L$ ). Similar graph was originally presented in Ref. [11].

As is shown in Fig. 6, which combines the discrete data points for $N=11$ to 19 , the dispersions are very clear for the three-body Pfaffian Hamiltonian $\mathcal{H}_{\mathrm{Pf}}$ (much less so for a pure Coulomb interaction $\mathcal{H}_{\mathrm{C}}$, but rather similarly for its modified version $\mathcal{H}_{1}$ - both not shown). As it has been discussed in detail in the original paper [11], the two dispersions are markedly different at short wave vectors, but - remarkably - converge to the same value in the limit of large wave vector $(k \lambda>2)$. It turns out that this degeneracy is not accidental [13], instead being a crucial property of the universality class of paired composite fermion states represented by the Pfaffian wave function, and it will be explained in the following section.

\section{Unpaired CF addition energy}

The energy of the bottom of the dispersion shown in Fig. 6 defines the addition energy of a composite fermion in the absence of other excitations, or the energy gap of the Pfaffian ground state for breaking composite fermion pairs. This gap is evidently non-zero, as required for an incompressible paired ground state. However, one of the consequences of the Ising fusion rules (2) is that a composite fermion can be added at a zero energy cost in the presence of two widely separated quasiparticles. This nontrivial prediction has been tested [11] for different model Hamiltonians used in Fig. 5, and indeed it has been confirmed even for the pure Coulomb repulsion - despite the lack of clearly developed dispersion in Fig. 5 or (not shown) of a clearly developed band of two-quasiparticle states for this realistic interaction.

The result of the calculation reported in [11] is plotted in Fig. 7, showing the independence of the 2-quasiparticle energy of the parity of the electron number $N$. It is worth mentioning that the space of two quasiholes (formed at flux $2 Q=2 N-2$ ) or two quasielectrons (at $2 Q=2 N-4$ ) contains the states with all possible angular momenta $L$, corresponding to different average $\mathrm{QH}-\mathrm{QH}$ or $\mathrm{QE}-\mathrm{QE}$ distances $d$. However, as the relation between $L$ and $d$ is affected by the presence of the neutral fermion, a direct comparison of the $2 \mathrm{QP}$ and $2 \mathrm{QP}+\mathrm{NF}$ energies (or $2 \mathrm{QE}$ and $2 \mathrm{QE}+\mathrm{NF}$ energies) is impossible. Hence, the energy $E$ shown in Fig. 7 represents an average over all space (all $L$-multiplets, weighted by $2 L+1$ ), thus including the configurations with both distant and nearby QHs (or QEs) alike. The lack of a significant parity effect in $E(N)$ points therefore not only to the vanishing of the neutral fermion addition energy in the presence of distant quasiparticles, but also to a rather small even/odd splitting for the nearby quasiparticles. To be more precise, a small even/odd energy difference is visible for finite $N$, but it disappears under extrapolation to the thermodynamic limit (with widely separated quasiparticles). Therefore, in terms of the underlying Ising conformal field theory, the nearly vanishing gap $E(N+1)-E(N)$, for even $N$, represents the degeneracy of the two possible distinct fusion channels $\mathbf{1}$ and $\psi$ in Eq. (2), for well separated vortices $\sigma$. By contrast, it is possible to focus on the configurations with nearby quasiparticles, realized for the largest angular momentum values of the low-lying eigenstates. In this case, we find a negative splitting, $E(N+1)-E(N)<0$, in the thermodynamic limit, indicating that the $\psi$-channel is energetically preferred over the identity 1-channel when vortices are in proximity (for details see Ref. [11]).

\section{Bipartite composite fermion model}

The above subtle features of the Pfaffian ground state and its excitation spectrum become evident in the recently proposed bipartite composite fermion model [13-15]. The Pfaffian wave function (4) can also be expressed as

$$
\Psi_{\mathrm{Pf}}=\mathcal{A} \prod_{i<j}\left(z_{i}-z_{j}\right)^{3} \prod_{i<j}\left(w_{i}-w_{j}\right)^{3} \prod_{i, j}\left(z_{i}-w_{j}\right),
$$

where the total number of electrons $N$ have been divided into two equally $(N / 2)$ populated partitions " $z$ " and " $w$ " (as in the "331" bilayer wave function [16]) and $\mathcal{A}$ is the antisymmetrizing operator (under exchanges among all $N$ fermions). The pairing nature of the above state clearly follows from the intrapartition correlations (de- 


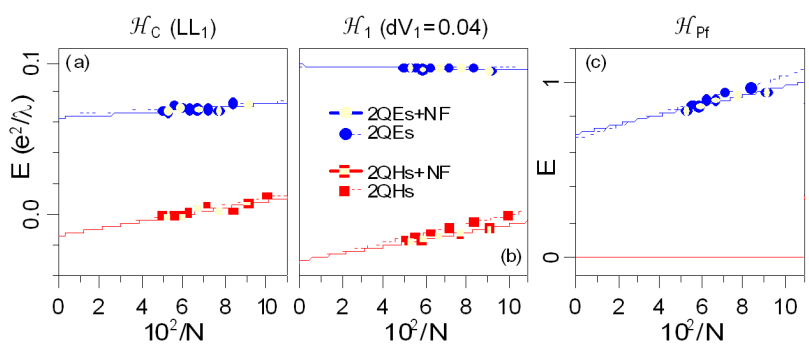

Fig. 7. The comparison of the total energies of a pair of quasiparticles (quasielectrons or quasiholes) created by insertion or removal of a single flux quantum to/from a state at the magnetic flux $2 Q=2 N-3$, with and without an additional neutral fermion (i.e., for an odd or even electron number $N$ ) for systems of different size and for different Hamiltonians of Fig. 5. The energies $E$ have been averaged over all 2-quasielectron or 2 -quasihole (and/or a neutral-fermion) states. The insensitivity of $E$ to the parity of $N$, i.e., to the presence or absence of the quasiparticles, is consistent with the expectation of zero neutral-fermion addition energy in the presence of two distant (non-Abelian) quasiparticles. After Ref. [11].

fined by the Jastrow exponent "3") being stronger than the interpartition ones (with exponent " 1 ").

The above inter- or intrapartition correlations can be reproduced by an appropriate flux attachment procedure equivalent to a bipartite composite fermion model with an effective magnetic field $B^{*}$ acting on each composite fermion partition separately. For example, for a Pfaffian ground state on a sphere, which results for $2 Q=2 N-3$ and an even $N$, each partition senses the effective magnetic flux $2 Q^{*}=2 Q-2(N / 2-1)-N / 2=N / 2-1$, consistent with its exact filling by $N / 2$ particles. In the following we shall also carefully examine application of this model to the Pfaffian excitations.

Let us allow for an arbitrary composite fermion number imbalance $\delta$ between the two partitions, thus introducing a pair of partition counts

$$
N_{ \pm}=\frac{N \pm \delta}{2}
$$

such that $N_{+}+N_{-}=N$ and $N_{+}-N_{-}=\delta \geq 0$. The imbalance $\delta$ has the same parity as $N$, which can be either even or odd. The effective flux acting on each partition is

$$
2 Q_{ \pm}^{*}=2 Q-2\left(N_{ \pm}-1\right)-N_{\mp} .
$$

Near the Landau level half-filling we also define an offset $s$ from the flux corresponding to the Pfaffian ground state

$$
2 Q=2 N-3+s .
$$

In attempt to identify the low-energy configurations, let us now consider a problem of filling two partitions "+" and "-" (of degeneracy $2 Q_{ \pm}+1$ ) with $N_{+}$and $N_{-}$composite fermions, respectively. Equation (8) can be conveniently rewritten in the following form:

$$
\eta_{ \pm} \equiv 2 Q_{ \pm}^{*}+1-N_{ \pm}=s \mp \delta,
$$

expressing the quasiparticle numbers $\eta$ in both partitions as a function of the offset $s$ and the partition imbalance $\delta$ (and independently of the electron number $N$ or flux $2 Q$ ). The convention is that positive and negative $\eta$ count the quasiholes and quasielectrons, respectively.

It has been conjectured [13] that the eigenstates of the many-electron Pfaffian Hamiltonian $\mathcal{H}_{\mathrm{Pf}}$ (and, possibly, also of a more realistic Hamiltonian yielding equivalent dynamics) are ordered in their interaction energy $E$ with respect to the total cyclotron energy of the corresponding configuration of composite fermions, denoted by $E^{*}$. In particular, the $E=0$ states of $\mathcal{H}_{\mathrm{Pf}}$ will be represented by the configurations with all composite fermions remaining in the lowest Landau levels in their respective partitions which, according to (10), requires

$$
\delta \leq s
$$

Let us consider each value of $s$ separately. The composite fermion energy $E^{*}$ will be measured in the units of the effective cyclotron energy $\hbar \omega_{\mathrm{c}}^{*}$ and counted from $N / 2$, so that $E=0$ corresponds to $E^{*}=0$.

For $s=0$, the zero-energy $(E=0)$ condition $(10)$ admits a single partition imbalance $\delta=0$, for which $\eta_{+}=\eta_{-}=0$, implying that in each partition the lowest Landau level is exactly filled by $N_{+}=N_{-}=N / 2$ composite fermions. This yields a unique (Pfaffian) state at $L=0$, which can be pictured as

\section{$-0000000000-0000000000-$}

For $s=1$, the $E=0$ states are possible for two distinct partition choices, corresponding to the imbalance values $\delta=0$ and 1 . They all represent a pair of quasiholes in the Pfaffian ground state. For $\delta=0$, Eq. (10) yields $\eta_{+}=\eta_{-}=1$, and the quasiholes appear in different partitions, which is pictured as

\section{$-0000000000-0000000000$}

For $\delta=1$, we get $\eta_{+}=0$ and $\eta_{-}=2$, with both quasiholes appearing in the same ("-") partition, pictured as

\section{$0000000000-000000000$}

The possible values of angular momentum $L$ result from addition rule for pairs of identical (composite) fermions, with the angular momenta of individual quasiholes given by $\ell_{ \pm}=Q_{ \pm}^{*}$. The result is $L=L_{\mathrm{MAX}}-2 j$, with $j=0,1, \ldots$ enumerates the pair states and $L_{\mathrm{MAX}}=$ $N / 2$ and $(N-1) / 2$ for $\delta=0$ and 1 , respectively.

As $\delta$ and $N$ always have equal parities, the two kinds of $E=0$ configurations (distinguished by the imbalance $\delta=0$ or 1 ; for the particular offset $s=1$, all representing two Pfaffian quasiholes) require different parities of $N$. Thus, for a fixed parity of $N$, there is only one kind of the $E=0$ configuration, with all states distinguished by 
an orbital quantum number $L$. This is consistent with the lack of topological degeneracy for two Pfaffian quasiholes.

For $s=2$, three distinct $E=0$ configurations can have $\delta=0,1$, or 2 . All of them contain four Pfaffian quasiholes. Importantly, the two corresponding to the even $\delta$ arise for the same (even) parity of $N$. Let us look at them in detail.

For $\delta=0$, from Eq. (10) we find $\eta_{+}=\eta_{-}=2$, with two quasiholes appearing in each partitions, pictured as

\section{$-0000000000-0000000000$}

For $\delta=2$, we have $\eta_{+}=0$ and $\eta_{-}=4$, with all quasiholes placed in the same ("-") partition, pictured as

\section{$-00000000000-000000000$}

Again, the allowed total angular momenta $L$ can be understood by applying addition rules to four quasiholes with appropriate individual angular momenta $\ell=Q^{*}$. We will not list here the whole spectrum, but it is important to observe that in a sufficiently large system the small values of $L$ corresponding to a large average quasihole separation will repeat for $\delta=0$ and 2. Hence, an identical spatial configuration of four quasiholes in a system with well-defined $N$ and $2 Q$ can be represented by two distinct quantum states - here distinguished by the imbalance $\delta$. This is pleasingly consistent with the known emergence of the topological degeneracy in a system of four Pfaffian quasiholes, implied by the Ising fusion rules (2).

The states corresponding to two or four quasielectrons can be identified analogously as the configurations with the minimum $E^{*}$ (except they no longer can have $\left.E^{*}=0\right)$. For example, for $s=-1$, the minimum composite fermion cyclotron energy $E^{*}=2$ can be achieved in two ways. For $\delta=0$, the two quasielectrons are evenly distributed between the partitions

\section{$\frac{0}{-0000000000}-0000000000$}

For $\delta=1$, they both appear in the same partition

\section{$000000000-000000000$}

By adding two individual quasielectron angular momenta $\ell=Q^{*}+1$ (note that each quasielectron is represented by a composite fermion in the second Landau level) one can readily predict the allowed values of $L=L_{\mathrm{MAX}}-2 j$ with $L_{\mathrm{MAX}}=N / 2$ and $(N-3) / 2$ for $\delta=0$ and 1 , respectively. In contrast to two-quasihole states defined unambiguously in the spectra of $\mathcal{H}_{\mathrm{Pf}}$ at $E=0$, the two-quasielectron states cannot be confidently identified in the $N$-electron spectra because they all have $E>0$. Nonetheless, for odd $N$, the value of $L_{\mathrm{MAX}}=(N-3) / 2$ indeed appears to agree with the spectra of $\mathcal{H}_{\mathrm{Pf}}$ [11]. However, for even $N$, the value of $L_{\mathrm{MAX}}=N / 2$ seems too high [11] (for an unknown reason).

Let us now return to the previous case of $s=0$ and look at the neutral modes. The corresponding states have $E^{*}=1$; they are possible for two different partition choices.

For $\delta=0$, they constitute the first excited band (above the $E^{*}=0$ Pfaffian ground state), in the form of an inter-Landau-level exciton, with both the quasielectron and the quasihole placed in the same partition (either "-" or "+"; their equivalence is guaranteed by antisymmetrization), which can be pictured as

\section{$-0000000000-0000000000$}

For $\delta=1$, the $E^{*}=1$ configurations constitute the lowest energy band, so one can use Eq. (10) to find $\eta_{+}=-1$ and $\eta_{-}=1$. This also corresponds to an inter-Landau-level exciton, but one with the quasielectron and the quasihole placed in different partitions ("+" and "-", respectively), which can be pictured as

\section{$-0000000000-000000000$}

The above pair of neutral modes with $\delta=0$ and 1 have been identified [13] as the "ordinary" and "topological" [15] composite fermion excitons, respectively. The latter name reflects the involved change in the electron number parity, and the resulting protection from self-annihilation at a constant $N$. The topological exciton has been further identified with the Majorana composite fermions [13].

The allowed angular momenta $L$ of both excitons can also be predicted from angular momenta addition for the constituent (distinguishable) quasiparticles: $L=2,3$, $\ldots, N / 2$ for $\delta=0$ and $L=3 / 2,5 / 2, \ldots, N / 2$ for $\delta=1$ (except for the absence of $L=1$ and $L=1 / 2$ states which results in a non-trivial way from antisymmetrization $[13,17])$.

Having introduced the composite fermion excitons, we can now revisit the many-quasiparticle states at $s \neq 0$. It can be readily observed that for a given total number of quasiparticles in the lowest-energy sector, $\eta_{+}+\eta_{-}=2 s$, the transitions between different values of imbalance $\delta$, corresponding to transferring quasiparticles between the two partitions, are realized by adding one kind of exciton and removing the other. In particular, the connection of four-quasiparticle configurations with the same parity of $\delta$ involves an even (but non-zero) number of topological excitons, which explains their topological distinction.

The bipartite composite fermion model gives natural insight [13] into several known properties of the Pfaffian state: (i) the odd-even effect [18] for the energy of a half-filled second Landau level as a function of the electron number $N$ - associated with the gap to add an unpaired composite fermion (topological exciton) at odd $N$; (ii) occurrence of two collective modes - understood as the ordinary and "trans" composite fermion 


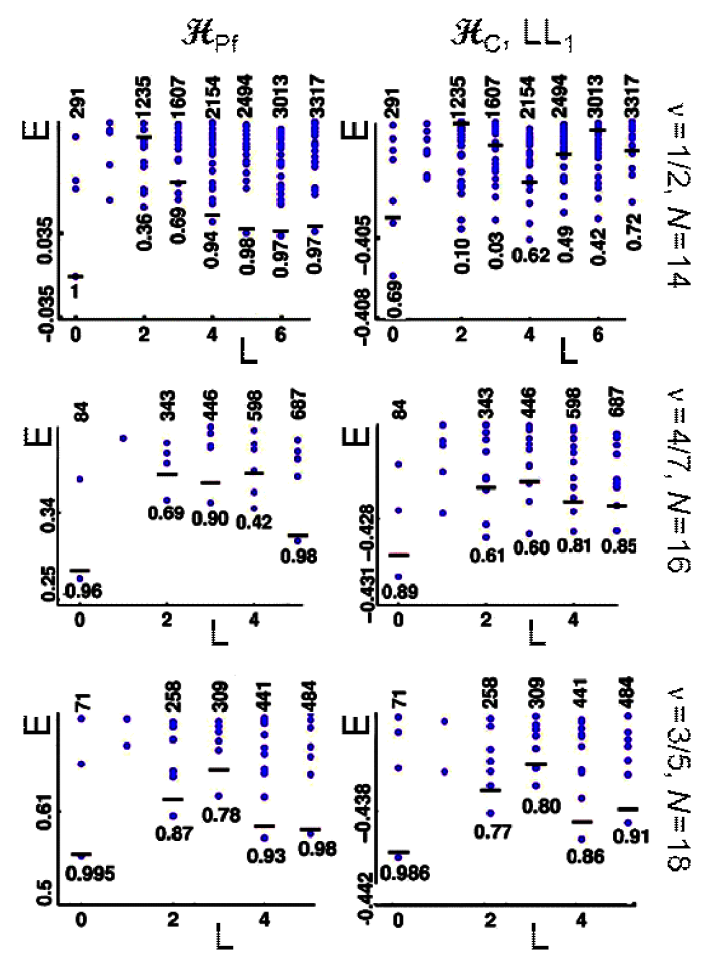

Fig. 8. The energy spectra of $N$ fermions on a sphere, with different interaction Hamiltonians: three-body contact repulsion $\left(\mathcal{H}_{\mathrm{Pf}}\right)$ and the Coulomb repulsion in the second Landau level $\left(\mathcal{H}_{\mathrm{C}}\right)$. The Coulomb energies are quoted in the units of $e^{2} / \lambda$. In consecutive rows, the magnetic monopole strengths $2 Q$ are chosen so as to yield $q=1,2,3$ filled composite fermion Landau levels in the bipartite model, corresponding to the indicated electron filling factors $\nu=1 / 2$ (Pfaffian), 4/7, and $3 / 5$. The labels at the low-energy states give their overlaps with the bipartite composite fermion wave functions, whose average energies are also shown with bars. The numbers above each column of data points indicate the space dimension at a given $L$. After Ref. [14].

excitons, and their degeneracy in the large wave vector limit - corresponding to a large quasielectron-quasihole separation within the exciton; (iii) degeneracy of wide two-quasiparticle states that are different in the number of electrons $N$ - connected by the quasiparticle transfer between the partitions; (iv) the Ising fusion rules almost self-evident upon the identification of fields $\mathbf{1}, \psi$, and $\sigma$ with the relevant composite fermion excitations: ordinary (cis-) exciton, topological (trans-) exciton, and a single quasiparticle, respectively.

The bipartite composite fermion model has also been generalized [14] to other filling factors. For example, the states at $\nu=2+4 / 7=18 / 7$ and $2+3 / 5=13 / 5$ have been accurately described by the bipartite states with two and three filled Landau levels in each partition, respectively. Importantly, these fractions are connected by the particle-hole conjugation (in the second Landau level) with $\nu=2+3 / 7=17 / 7$ and $2+2 / 5=12 / 5$, at which the quantum Hall effect has been observed [19].
Numerical evidence for the validity of the bipartite composite fermion model has been discussed in detail previously $[13,14]$. Below, in Fig. 8 , we present only a few examples of the energy spectra of $\mathcal{H}_{\mathrm{Pf}}$ and $\mathcal{H}_{\mathrm{C}}$, for the $N$-electron systems representing filling factors $\nu=1 / 2$, $4 / 7$, and $3 / 5$ (corresponding to $q=1,2,3$ filled Landau levels in each partition). Especially for $\mathcal{H}_{\mathrm{Pf}}$ description of both the ground states and the excited magneto-roton bands is accurate, and the issue of adiabatic continuity between $\mathcal{H}_{\mathrm{Pf}}$ the $\mathcal{H}_{\mathrm{C}}$ has been explored in Ref. [13].

\section{Conclusion}

We have characterized the $\nu=5 / 2$ fractional quantum Hall state formed in a half-filled second Landau, which is believed to be a realization of the so-called "Pfaffian" ground state with non-Abelian quasiparticles. We have described the excitations of the Coulomb spectrum and demonstrated a qualitative signature of pairing in this state: the existence of a well-defined excitation mode occurring for odd numbers of particles. We have further reviewed a recently developed model describing the particular form of pairing correlation in the partially filled second Landau level in terms of multiple partitions of non-interacting composite fermions. We have explored in detail the half-filled case $(\nu=5 / 2)$. As we have shown, this model captures in an elegant way several exotic properties of the Pfaffian phase, including the emergence of topological degeneracy of multi-quasiparticle configurations (an essential requirement for non-Abelian braid statistics).

\section{Acknowledgments}

We acknowledge financial support from the EU Marie Curie grant No. PCIG09-GA-2011-294186 (A.W.), DOE - under grant No. DE-SC0005042 (G.J.S. and J.K.J.), Trinity Hall Cambridge (G.M.), the Bolyai Fellowship of the Hungarian Academy Sciences (C.T.), and EPSRC under EP/F032773/1 (N.R.C.). The calculations have been carried out in Penn State Research Computing and Cyberinfrastructure and Wrocław Centre for Networking and Supercomputing.

\section{References}

[1] D.C. Tsui, H.L. Störmer, A.C. Gossard, Phys. Rev. Lett. 48, 1559 (1982).

[2] R.B. Laughlin, Phys. Rev. Lett. 50, 1395 (1983).

[3] J.K. Jain, Phys. Rev. Lett. 63, 199 (1989).

[4] F.D.M. Haldane, Phys. Rev. Lett. 51, 605 (1983).

[5] A. Stern, Ann. Phys. 323, 204 (2008); C. Nayak, S.H. Simon, A. Stern, M. Freedman, S. Das Sarma, Rev. Mod. Phys. 80, 1083 (2008).

[6] A.Yu. Kitaev, Ann. Phys. 303, 2 (2003); S. Das Sarma, M. Freedman, C. Nayak, Phys. Rev. Lett. 94, 166802 (2005). 
[7] G. Moore, N. Read, Nucl. Phys. B 360, 362 (1991); M. Greiter, X.-G. Wen, F. Wilczek, Phys. Rev. Lett. 66, 3205 (1991); N. Read, D. Green, Phys. Rev. B 61, 10267 (2000).

[8] R. Willett, J.P. Eisenstein, H.L. Stormer, D.C. Tsui, A.C. Gossard, J.H. English, Phys. Rev. Lett. 59, 1776 (1987); M. Dolev, M. Heiblum, V. Umansky, A. Stern, D. Mahalu, Nature 452, 829 (2008); C.R. Dean, B.A. Piot, P. Hayden, S. Das Sarma, G. Gervais, L.N. Pfeiffer, K.W. West, Phys. Rev. Lett. 100 , 146803 (2008).

[9] G. Möller, S.H. Simon, Phys. Rev. B 77, 075319 (2008); A. Wójs, Phys. Rev. B 80, 041104(R) (2009); M. Storni, R.H. Morf, S. Das Sarma, Phys. Rev. Lett. 104, 076803 (2010).

[10] R.H. Morf, Phys. Rev. Lett. 80, 1505 (1998); A.E. Feiguin, E.H. Rezayi, K. Yang, C. Nayak, S. Das Sarma, Phys. Rev. B 79, 115322 (2009); A. Wójs, G. Möller, S.H. Simon, N.R. Cooper, Phys. Rev. Lett. 104, 086801 (2010).

[11] G. Möller, A. Wójs, N.R. Cooper, Phys. Rev. Lett. 107, 036803 (2011).

[12] P. Bonderson, A.E. Feiguin, C. Nayak, Phys. Rev. Lett. 106, 186802 (2011).
[13] G.J. Sreejith, A. Wójs, J.K. Jain, Phys. Rev. Lett. 107, 136802 (2011).

[14] G.J. Sreejith, C. Tóke, A. Wójs, J.K. Jain, Phys. Rev. Lett. 107, 086806 (2011).

[15] T.H. Hansson, M. Hermanns, S. Viefers, Phys. Rev. B 80, 165330 (2009); T.H. Hansson, M. Hermanns, N. Regnault, S. Viefers, Phys. Rev. Lett. 102, 166805 (2009).

[16] B.I. Halperin, Helv. Phys. Acta 56, 75 (1983).

[17] G. Dev, J.K. Jain, Phys. Rev. Lett. 69, 2843 (1992).

[18] H. Lu, S. Das Sarma, K. Park, Phys. Rev. B 82, 201303 (2010).

[19] W. Pan, J. Xia, V. Shvarts, D. Adams, H. Stormer, D. Tsui, L. Pfeiffer, K. Baldwin, K. West, Phys. Rev. Lett. 83, 3530 (1999); J.S. Xia, W. Pan, C.L. Vicente, E.D. Adams, N.S. Sullivan, H.L. Störmer, D.C. Tsui, L.N. Pfeiffer, K.W. Baldwin, K.W. West, Phys. Rev. Lett. 93, 176809 (2004); H.C. Choi, W. Kang, S. Das Sarma, L.N. Pfeiffer, K.W. West, Phys. Rev. B 77, 081301(R) (2008). 